\title{
Controle automatizado de casas de vegetação: Variáveis climáticas e fertigação
}

\author{
Bárbara J. Teruel ${ }^{1}$
}

\section{RESUMO}

A aplicação de técnicas de automação e controle em casas de vegetação teve início nos anos 50, com o controle da temperatura através de termostatos; desde então, as mudanças tecnológicas têm abarcado todos os aspectos que compõem o sistema, do projeto estrutural até aspectos relacionados à sustentabilidade. Dentre os parâmetros que devem ser controlados, a temperatura e umidade relativa do ar, a radiação solar, a concentração de $\mathrm{CO}_{2}$, a ventilação e a fertigação têm tido uma evolução significativa, dos quais se apresenta uma panorâmica através da revisão do estado da arte de trabalhos publicados nessas temáticas, nos últimos anos. $\mathrm{Na}$ atualidade, temas relacionados com a redução do impacto ao meio ambiente e a produção eficiente e com qualidade, estão levando as casas de vegetação ao caminho da Agricultura de Precisão.

Palavraschave: temperatura, umidade relativa, concentração de $\mathrm{CO}_{2}$, ventilação, solução nutritiva

\section{Automatized control in greenhouses: Climatic variables and fertigation}

\begin{abstract}
The application of techniques of automation and control in greenhouses began in the years 50 , starting with the control of temperature through thermostats. Since then, technological changes have covered all aspects that make up the system, from constructional features up to aspects of sustainability. The control of parameters sulh as, temperature and relative humidity, solar radiation, the concentration of $\mathrm{CO}_{2}$, ventilation and fertigation, had significant development, from which it provides a review of published works about these themes in recent years. Currently, issues related to reducing the impact to the environment and efficient production and quality, are taking the greenhouses towards Precision Agriculture.
\end{abstract}

Key words temperature, relative humidity, $\mathrm{CO}_{2}$ concentration, ventilation, nutrient solution 


\section{INTRODUÇÃO}

O sucesso da agricultura moderna está relacionado, de maneira intrínseca, com o gerenciamento eficiente da produção, em todas as suas formas, visando à qualidade do produto e eficiência do processo com a redução dos custos na busca de uma relação custo-benefício adequada. Particularmente, o cultivo em casas de vegetação já não é mais tratado como um sistema tradicional, tendo evoluído rapidamente, aplicando-se instrumentação, controle automático e tecnologias de informação, em busca da adaptação às demandas e exigências dos mercados consumidores.

A aplicação da automação pode contribuir com a agricultura para a melhoria da qualidade, a redução das perdas, o aumento da produtividade, a redução dos custos e diminuição do tempo de retorno do investimento, planejamento e tomada de decisão assim como na diminuição do impacto ao meioambiente, facilitando o trabalho e aumentando a qualidade de vida do produtor, visando a uma competitividade maior.

Algumas soluções já disponíveis para aplicação em casas de vegetação, eram consideradas distantes há alguns anos; exemplo disto é a aplicação de sistemas híbridos, de inteligência artificial, determinados tipos de sensores, aplicativos de supervisão e interfaces homem-máquina, transmissão de dados a distância, algoritmos de controle com base em modelos matemáticos que simulem o sistema, incorporando parâmetros biológicos, físicos e químicos, além dos balanços de calor e massa em função da variação das propriedades térmicas e físicas e as variáveis climáticas. O controle climático, por exemplo, já está sendo usado para controlar a temperatura do solo, a fim de induzir a floração de algumas espécies.

Observa-se, também, que os sistemas e processos agrícolas vêm sendo tratados com uma visão multidisciplinar no âmbito da ciência, incorporando as áreas da Física, da Engenharia Mecânica, da Eletrônica, do Controle e Automação e da Engenharia Química abrindo, assim, perspectivas para o desenvolvimento e aplicação das bases da Agricultura de Precisão, com visão de sistema ou cadeia (Sigrimis \& King, 2000; Stanford, 2000).

Em particular, a automatização de casas de vegetação permite um controle mais seguro e preciso do processo reduzindo a mão-de-obra, a ocorrência de doenças nas plantas, favorecendo o aumento da qualidade e produtividade, ao mesmo tempo em que podem ser obtidos conjuntos de dados e informações do processo, permitindo e facilitando a análise, gerando subsídios para a tomada de decisão, mas embora com todas essas vantagens, para que os benefícios possam ser garantidos, os sistemas de medição e controle em casas de vegetação necessitam de respostas rápidas e precisas, de instrumentos sensíveis e estáveis, assim como a aquisição de dados em qualquer instante de tempo.

Embora as vantagens da aplicação de tecnologias de controle automático sejam evidentes, em estudo de Vischi Filho (2002), sobre o estado das tecnologias em casas de vegetação para flores, foram constatados diferentes graus de automação havendo a necessidade do controle de parâmetros, como a temperatura e umidade relativa do ar, luminosidade, radiação solar, ventilação, aquecimento e resfriamento.

A temperatura, a iluminação e a concentração de $\mathrm{CO}_{2}$, por exemplo, influenciam o processo de produção vegetal, propiciando o desenvolvimento adequado quando mantidos os limites estabelecidos ou provocando a morte das plantas, se as recomendações não são atendidas (Cansado \& Saraiva, 2003). Uma ampla discussão sobre a importância do controle da irrigação, da temperatura do ar e da radiação solar, em casas de vegetação, é apresentada por Beltrão et al. (2002).

Apresenta-se uma revisão e discussão sobre a aplicação de técnicas de instrumentação, controle automático e tecnologias de informação em casas de vegetação. A revisão foi estruturada em tópicos que incluíam definições, sensores e critérios para a implantação de malhas de controle além de resultados de pesquisas desenvolvidas para o controle e medição de variáveis que interferem no processo, dentre elas as climáticas, a concentração de $\mathrm{CO}_{2}$, a ventilação, a fertigação e a aplicação da solução nutritiva.

\section{CASAS DE VEGETAÇÃO}

Podem-se definir as casas de vegetação não apenas como uma estrutura coberta e abrigada artificialmente, para diferentes tipos de plantas e cultivos, protegendo-os contra os agentes meteorológicos externos; mas também como um meio controlado no qual o crescimento da planta depende de fatores como a água, a iluminação, os fertilizantes, o oxigênio e o dióxido de carbono $\left(\mathrm{CO}_{2}\right)$ (Beltrão et al., 2002; Vischi Filho, 2002).

Algumas tecnologias, dentre elas telas de sombreamento, nebulizadores e iluminação suplementar, permitem que variáveis como a temperatura, a luz, a concentração de $\mathrm{CO}_{2}$, o volume de ar, de substrato e de nutrientes, possam ser automaticamente controladas contribuindo para o aumento do valor comercial dos produtos e economia de energia e de insumos (Bliska Jr. \& Honório, 1996).

\section{Sistemas de controle para casas de vegetação}

Um sistema de controle está baseado na combinação de hardware e software, devendo ser robusto e integrado ao processo da casa de vegetação considerando-se tanto as estratégias de controle como os sensores adequados ao processo e parâmetros a serem mensurados e sistemas de aquisição e processamento de dados, em tempo real. Para casas de vegetação, malhas de controle automático com realimentação podem ser uma solução adequada, devido às vantagens que oferecem, em termos da resposta e atuação. O sistema de medição e as técnicas de instrumentação devem ser, sempre que possível, não-destrutivos, dando preferência às medidas diretas, fazendo-se as medições não apenas em uma planta e, sim, de um grupo delas, obtendo-se amostragem representativa do processo (Helmer et al., 2005).

\section{Sensores}

Os sensores compõem um elemento importante nas malhas de controle, visto que fornecem os dados que serão usados para definir a atuação no processo de uma casa de vege- 
tação mas, tanto a seleção como as técnicas de medição devem ser tratadas com cuidado, evitando-se a ocorrência de erros que afetam sensivelmente a precisão e efetividade das estratégias de controle. Neste sentido, Helmer et al. (2005), discutiram como a influência das condições climáticas, dentre outros fatores inerentes ao processo nas casas de vegetação, pode provocar erros grosseiros. Os autores exemplificaram esta influência citando os sensores de medição da umidade do solo, cujo princípio de funcionamento está baseado na condutividade elétrica de pontas de medição, produzindo-se um sinal elétrico quando há variação de umidade próximo do ponto de medição. As medidas feitas por estes instrumentos são afetadas significativamente pela variação de temperatura e a quantidade de sais dissolvidos nos fertilizantes do solo, na região da inserção da ponta de medição e no instante específico e que o valor é medido, no entanto, isto não significa que seja uma variação significativa no processo ao longo do tempo.

Com base nesta discussão, a seleção dos sensores deve ser criteriosa atendendo às exigências e condições do processo, às características estáticas (como range, precisão, exatidão, repetivividade e sensibilidade), e às características dinâmicas, como o tempo de resposta devendo, também, adequarse aos fenômenos e características dos processos e instalações. É conveniente que os instrumentos sejam selecionados, atendendo também às limitações operacionais da casa de vegetação, para que não obstruam nem dificultem o funcionamento da instalação. Muitas vezes, os instrumentos apenas medem a variável definida em uma região específica da planta ou da casa de vegetação, havendo necessidade de grande quantidade de instrumentos para mapear o comportamento de toda a planta ou processo; exemplo disto é a medição da abertura de estômatos, que permite o conhecimento do processo de fotossínteses; se esta medida é tomada em um ponto localizado e/ou uma posição única e específica, não deve ser utilizada como critério para expressar o comportamento da planta a menos que várias medidas em diversas localizações na planta sejam feitas.

Constata-se haver uma variedade de tipos de sensores para a medição das variáveis que interferem nos processos das casas de vegetação, como as climáticas e do solo porém o comportamento fisiológico das plantas raramente é mensurado, sendo esta uma fonte de informações importantes para estabelecer as estratégias de controle; por exemplo, a medição da taxa de transpiração das plantas fornece dados mais representativos do processo fisiológico que os obtidos através de simulações e modelos matemáticos (Helmer et al., 2005).

Instrumentos como os transdutores variáveis lineares (Linear Variable Differential Transforme, LVDT), tem sido usados para quantificar a taxa de crescimento dos frutos; no entanto, o funcionamento e a estabilidade dos LVDTs requerem de estrutura específica e alimentação elétrica estável (Cohen et al., 1998). Os anemômetros sônicos, por exemplo, usados geralmente em estações meteorológicas, podem ser utilizados em casas de vegetação para a medição da velocidade do ar, tendo em vista a sensibilidade, a resolução, a taxa de amostragem e a precisão, obtendo-se dados confiáveis que podem servir para a validação de simulações e modelos matemáticos. A título de exemplo, Reichrath \& Davies (2002), usaram as medições da velocidade do ar realizadas com um anemômetro sônico para a validação da simulação do escoamento do ar no interior de uma casa de vegetação, e a ferramenta computacional CFD (Computational Fluid Dynamics), e obtiveram boa aproximação entre os resultados teóricos e os experimentais. Como destacado por Norton et al. (2007), as características de desempenho desses instrumentos, aliadas à simulação através do CFD, podem ser úteis para estudar o comportamento de regiões das casas de vegetação onde há dificuldade de instalação de sensores.

Recomendações sobre a seleção e o uso de sensores em casas de vegetação são apresentadas pela Associação Nacional de Fabricantes de Casas de Vegetação (National Greenhouse Manufacturers Association) (NGMA, 2007).

\section{Critérios para a implantação de sistemas de controle em casas de vegetação}

Antes da definição das estratégias e desenho das malhas de controle para casas de vegetação, deve-se realizar um estudo preliminar a fim de que a implantação contribua para a obtenção da relação custo-benefício adequada, visando à otimização, racionalização e qualidade do processo e do produto, observando-se todas as funcionalidades de gestão das variáveis envolvidas. Deve-se, também, contemplar a identificação das variáveis mensuráveis e as que serão controladas definindo-se as estratégias de controle e hardwares e softwares a serem utilizados.

\section{Identificação das variáveis mensuráveis}

Deve ser a primeira etapa do estudo preliminar, definindo-se as variáveis que interferem no processo e que devem ser medidas e/ou monitoradas. A partir dessas definições poder-se-á definir os tipos de instrumento e sistemas de aquisição dos dados.

Segundo a NGMA (2007), os parâmetros e processos que devem ser controlados em casas de vegetação, são: a temperatura, a ventilação (ventiladores, dampers), a umidade relativa do ar (nebulizadores, desumificadores), a iluminação (lâmpadas, cortinas, janelas), a concentração de $\mathrm{CO}_{2}$ (injeção e/ou extração), a carga térmica interna, a irrigação (bombas, válvulas), os tratamentos químicos, a aplicação de nutrientes (mistura, injeção) e o abastecimento de água (bombas, válvulas).

Algumas vezes, variáveis importantes para o processo e que não podem ser medidas de forma contínua com os sensores da malha de controle, são passíveis de controle através de computadores; por exemplo, a quantidade de fertilizante na solução nutritiva é de difícil mensuração contínua; então, um computador pode ser usado para aplicar quantidades predeterminadas de nutrientes em intervalos pré-especificados, atuando como temporizador e não em função de um elemento de controle da malha.

\section{Identificação das variáveis a controlar}

São as variáveis que afetam diretamente o sistema de produção das casas de vegetação e que, também, são o alvo do 
sistema de controle. As variáveis que não podem ser controladas, mas afetam o processo, devem ser consideradas distúrbios no sistema de controle enquanto as que não podem ser ajustadas a um set point, porém podem ser úteis na estratégia de controle, são passíveis de serem consideradas variáveis intermediárias.

Algumas das variáveis que devem ser medidas e monitoradas em casas de vegetação, assim como a relação com as variáveis que devem ser controladas para garantir a qualidade do processo, se mostram na Tabela 1.

Tabela 1. Variável a mensurar em casas de vegetação e relação com os fenômenos

\begin{tabular}{|c|c|}
\hline Variável & Relacionada com \\
\hline $\begin{array}{l}\text { Temperatura do ar no interior da } \\
\text { casa de vegetação }\end{array}$ & Funções metabólicas das plantas. \\
\hline $\begin{array}{l}\text { Temperatura do ar externo à casa de } \\
\text { vegetação }\end{array}$ & $\begin{array}{l}\text { Ventilação e transferência de calor por } \\
\text { condução e convecção. }\end{array}$ \\
\hline $\begin{array}{l}\text { Umidade relativa do ar no interior da } \\
\text { casa de vegetação }\end{array}$ & $\begin{array}{l}\text { Transpiração e mecanismos de controle } \\
\text { térmico das plantas. }\end{array}$ \\
\hline $\begin{array}{l}\text { Umidade relativa do ar externo à casa } \\
\text { de vegetação }\end{array}$ & $\begin{array}{l}\text { Umidade relativa interna através da } \\
\text { ventilação. }\end{array}$ \\
\hline $\begin{array}{l}\text { Radiação solar externa à casa de } \\
\text { vegetação }\end{array}$ & $\begin{array}{l}\text { Processos de fotossínteses; aumento da } \\
\text { carga térmica. }\end{array}$ \\
\hline Velocidade do ar & $\begin{array}{l}\text { Diferença de potencial de água ar-folha; } \\
\text { ar úmido com propriedades estáveis. }\end{array}$ \\
\hline $\begin{array}{l}\text { lluminação no interior da casa de } \\
\text { vegetação }\end{array}$ & $\begin{array}{l}\text { Processos de fotossínteses; crescimento } \\
\text { e desenvolvimento vegetal; estiolamento } \\
\text { das plantas }\end{array}$ \\
\hline Concentração de $\mathrm{CO}_{2}$ & $\begin{array}{l}\text { Crescimento das plantas; } \\
\text { desenvolvimento do sistema radicular e } \\
\text { absorção de nutrientes, quando este é } \\
\text { adicionado à água. }\end{array}$ \\
\hline
\end{tabular}

Fonte: Adaptado de Zazueta et al. (1991)

\section{Estratégias de controle}

A definição das estratégias de controle é uma etapa significativa no dimensionamento das malhas. A estratégia mais simples é a de conectar os sensores diretamente aos atuadores, a exemplo dos termostatos. Outro nível de malhas de controle se baseia em sistemas de múltiplos estágios, por exemplo, para o resfriamento os ventiladores podem ser acionados, um por vez, na medida em que a temperatura aumenta; malhas de controle também podem incluir algoritmos com base em regras de decisão, como a lógica fuzzy.

Outras estratégias de controle podem ser baseadas não apenas nos sinais das variáveis controladas como, também, em dados históricos do processo conhecendo-se, por exemplo, a taxa de transferência de calor e massa e a dinâmica do processo; são os chamados sistemas de controle dinâmicos, cuja estratégia está baseada no conhecimento do fenômeno físico, razão pela qual ocorrem, nos processos das casas de vegetação, vários fenômenos complexos e que dependem de diversos fatores, térmicos, físicos, biológicos e químicos que são também intrinsecamente dinâmicos e regidos por equações diferenciais em função do tempo que, se corretamente combinadas, podem gerar modelos para o estabelecimento de estratégias adequadas de controle.

As falhas de sensores e atuadores em malhas de controle também têm uma importância significativa na efetividade do sistema; por isto, métodos de detecção e identificação dessas falhas são uma ferramenta necessária, como discutido por Linker et al. (2000). Os autores apresentaram uma ferramenta validada com as medições de variáveis climáticas de casas de vegetação operando comercialmente. O método combina sistemas híbridos e modelos de redes neurais com robustos sistemas de detecção, identificação e da teoria de sistemas não-lineares. As falhas dos sensores e dos atuadores foram detectadas assim como o estresse hídrico das plantas, estimando-se os custos provocados pelas falhas.

\section{Identificação do hardware e software}

Essas definições devem ser feitas antes da aquisição e instalação dos sensores nas casas de vegetação. A diferença, por exemplo, entre as malhas de controle com realimentação e os sistemas de controle através de computadores, é a flexibilidade que os computadores oferecem. Do ponto de vista do usuário, o uso de computadores possibilita flexibilidade e facilidade do uso de interfaces de entrada e saída, podendo especificar detalhes específicos dos algoritmos de controle no software, assim como a expansão da quantidade de variáveis mensuradas (entradas do sistema) e das saídas da malha de controle (saídas para atuação), em função da necessidade e do crescimento da produção. A seleção do hardware deve ser compatível com o software a ser utilizado, considerando-se, também, a confiabilidade, o conhecimento prévio do equipamento e da tecnologia, a possibilidade de suporte técnico, a manutenção e os custos (Zazueta et al., 1991).

Controle da concentração de $\mathrm{CO}_{2}$, umidade relativa do ar, radiação solar, ventilação, solução nutritiva e fertigação

Neste tópico serão discutidos o estado da arte do desenvolvimento e a aplicação de tecnologias e sistemas automatizados em casas de vegetação, visando ao controle das variáveis que têm interferência no processo, dentre elas as climáticas, a concentração de $\mathrm{CO}_{2}$, a ventilação, a solução nutritiva e a fertigação.

\section{Temperatura}

A temperatura é uma das variáveis que devem ser mensuradas visando ao controle, devido à sua influência nas funções metabólicas das plantas nas casas de vegetação. A temperatura é um parâmetro que varia significativamente com o tempo, sendo afetada por uma série de outras variáveis e fatores. Desta forma e dependendo das estratégias estabelecidas, será necessário um ajuste dos parâmetros de sintonia dos controladores em função da dinâmica do processo, que poderia ser atendido usando-se um sistema de controle adaptativo.

Os aspectos relacionados ao crescimento das plantas e a influência do controle das variáveis climáticas, dentre elas a temperatura, devem ser tomados em consideração pois, como mostrado por Straten et al. (2000), as características de cada cultura, a falta de conhecimento das respostas a diferentes tratamentos e respostas fisiológicas, podem levar à separação das responsabilidades do sistema de controle tendo efeito a curto e longo prazos, na qualidade da produção. 
O controle da temperatura tem sido feito aplicando-se uma variedade de técnicas, dentre elas o controle linear quadrático (LQ), através de controladores PID (Proporcional + Integral + Derivativo), controladores PI (Proporcional + Integral) e malhas com retroalimentação (feedback control), que cada vez vêm sendo mais usadas.

A aplicação de controladores PID para o controle das variáveis climáticas tem sido ampla em casas de vegetação mas não se tem mostrado como a melhor opção, pelas limitações que apresenta em relação à sintonia e ao número de entradas e saídas. Um algoritmo para o controle preditivo da temperatura do ar em casas de vegetação, com múltiplas entradas e saída (MIMO) e linearização on-line, é apresentado por El Ghoumari et al. (2005), mostrando ótimo desempenho do sistema de controle comparado com o controle PID e com o controle adaptativo PI, obtendo-se economia de energia no processo.

Malhas de controle com retroalimentação pseudoderivativa (Pseudo-Derivative-Feedback, PDF), se mostraram mais vantajosas se comparadas com o controle PI, tendo menor tempo de atraso, como mostrado por Setiawan et al. (2000).

Um sistema de controle no qual se usaram, como variável de entrada, as medidas da temperatura em diversas localizações de uma casa de vegetação, e um modelo para a predição do aquecimento e resfriamento do ar, em diferentes períodos de tempo, foram apresentados por Arvanitis et al. (2000), mostrando resultados confiáveis; os autores apresentam, também, uma ampla discussão sobre as vantagens desse tipo de sistema de controle de temperatura em casas de vegetação.

A aplicação de modelos preditivos tem a vantagem de prover o sistema de capacidade de reagir perante perturbações, evitando atrasos na resposta e atuação. Os algoritmos de controle para casas de vegetação devem utilizar modelos que descrevam o processo de desenvolvimento das plantas ao longo do tempo, podendo ser obtidos através de métodos determinísticos ou estocásticos, atendendo às não linearidades e dinâmica dos processos nessas instalações. As não-linearidades representam grande desafio dos processos na agricultura e vários estudos têm tratado de modelar e obter as respostas a partir de modelos lineares, introduzindo erros provocados pelas aproximações e considerações adotadas.

Buscando superar as limitações desses sistemas, Coelho et al. (2005) apresentaram um modelo de controle preditivo da temperatura do ar no interior de uma casa de vegetação, aplicando algoritmos de otimização e obtendo bons resultados se comparados com os obtidos através de um algoritmo genético e programação quadrática sequencial. Piñón et al. (2005) indicaram um sistema de controle de temperatura com combinação de controle linear preditivo (Feedback Linearization, FL) e controle preditivo (Model Predictive Control, MPC). O processo da casa de vegetação foi tratado como nãolinear, com uma entrada e saída (MIMO) e submetido a perturbações externas. Com base nos resultados obtidos na otimização das não-linearidades, as rápidas respostas e o tempo computacional, os autores recomendam referidas técnicas para a aplicação em sistemas comerciais. Herrero et al. (2007) apresentam um sistema de identificação de modelos não-lineares para o controle robusto das variáveis climáticas, dentre elas a temperatura, com base em equações de primeira ordem e na estimativa dos parâmetros (Feasible Parameter Set, FPS), aplicando otimização multimodal com um número infinito de constituintes do FPS.

A eficiência energética em casas de vegetação, em função da diminuição do consumo de energia elétrica, provocado pelo controle da temperatura do ar, vem sendo tratado e discutido nos último anos, como mostra o trabalho desenvolvido por Sigrimis et al. (2000b). Os autores desenvolveram um sistema de controle da temperatura da instalação através de modelos de simulação e a configuração de diferentes condições, através de um supervisório desenvolvido em SCADA (Supervisory Control And Data Acquisition). Os resultados foram comparados com dados experimentais mostrando-se promissores.

\section{Concentração de $\mathrm{CO}_{2}$}

A concentração de $\mathrm{CO}_{2}$ é um parâmetro com relativa dificuldade para ser medido e controlado, pois depende da ventilação da instalação e da fisiologia das plantas. Constata-se que a ventilação e a concentração de $\mathrm{CO}_{2}$ raramente são controladas simultaneamente, mantendo-se o controle da temperatura até certo valor de set point, após o qual são ligados os ventiladores. Se a medição da concentração de $\mathrm{CO}_{2}$ em casas de vegetação não é cuidadosa, ocorram erros que levam ao estabelecimento de estratégias de controle insatisfatórias e, por consequência, a alteração do processo e qualidade dos produtos, como destacado por Roy et al. (2002).

Outra questão a se destacar é a injeção de $\mathrm{CO}_{2}$, que deve ser realizada com cuidado, sobretudo em climas tropicais, quando também há necessidade de ventilação. O volume de $\mathrm{CO}_{2}$ injetado deve ser misturado homogeneamente com o ar no interior da instalação mas, como em geral a área da casa de vegetação é grande, caso a amostragem das medições seja limitada, haverá maior incidência de erros na atuação do sistema de controle. Uma recomendação para a diminuição desta fonte de erro é o acionamento de ventiladores auxiliares facilitando a homogeneização da mistura de ar e $\mathrm{CO}_{2}$. Recomenda-se que, sempre que possível, os sensores sejam dispostos em várias localizações da instalação permitindo que os dados obtidos nas medições sejam representativos do processo na casa de vegetação.

A medição da concentração de $\mathrm{CO}_{2}$, utilizando-se analisadores de gases, não tem mostrado ser suficientemente precisa, estando entre as prováveis fontes de erro; além daquelas introduzidas pelo próprio sistema de medição, a infiltração de ar em regiões diferentes à que está sendo medida; a concentração não uniforme de $\mathrm{CO}_{2}$ na mistura de ar e a abertura das janelas durante as medições (Roy et al., 2002); no entanto, e segundo Norton et al. (2007), se houver injeção contínua do gás poderão ser obtidas medições mais precisas mas, como há necessidade da integração e otimização das variáveis físicas e biológicas que interferem nos processos das casas de vegetação, a utilização de medidas experimentais aliada a modelos matemáticos para a simulação da concentração de $\mathrm{CO}_{2}$, se tem mostrado viável, obtendo-se resultados com boa aproximação e tornando 
uma ferramenta útil para o controle dos ambientes, como discutido por Bartzanas et al., (2004).

O processo de respiração, tal como o crescimento das plantas é um processo dinâmico, com respostas que variam ao longo do tempo e que tem influência no controle da concentração de $\mathrm{CO}_{2}$. Pensando nesta relação, um algoritmo representando e correlacionando o controle da concentração de $\mathrm{CO}_{2}$ com o peso das plantas, foi desenvolvido por Jin \& Hong (1993); já Trigui et al. (1993), utilizaram o conhecimento prévio de casas de vegetação e propuseram o controle dinâmico através de um modelo de transpiração e gerenciamento heurístico, com base na transpiração acumulada em 24 h, em função do crescimento das plantas e do déficit de pressão de vapor na superfície das folhas.

Recentemente, na tentativa de se obter respostas mais precisas para atuação do sistema de controle, tem-se feito a aplicação de técnicas computacionais de simulação fluidodinâmica, através da ferramenta computacional CFD (Computational Fluid Dynamics). As casas de vegetação, estilo parreira, também têm sido estudadas aplicando-se CFD. Referidas instalações são muito comuns em países do Mediterrâneo e da África do Norte e, embora construídas de forma empírica, com estrutura relativamente simples, de baixo custo, fornecem proteção às plantas da chuva e o vento e no inverno funcionam como coletor solar mas embora se constate a aplicação das técnicas de simulação e as ferramentas do CFD, para se entender o comportamento e a influência da concentração de $\mathrm{CO}_{2}$, é premente a necessidade de continuidade das pesquisas nesta direção eliminando-se as limitações que ainda persistem, entre elas a de não se considerar a influência da radiação solar incidindo-se em erros da ordem de $12 \%$ ou, então, a falta de validação de modelos de simulação propostos na literatura e outros que não têm potencial de aplicação para fins comerciais. A consideração da inércia térmica é necessária para que haja um efetivo controle do processo já que a influência da radiação solar faz com o que o sistema tenha comportamento transiente tornando as respostas dinâmicas (Norton et al., 2007).

\section{Umidade relativa do ar}

O controle da umidade relativa do ar durante o crescimento das plantas é oportuno, devido à sua influência na prevenção de doenças, na carga térmica, seja de aquecimento ou de resfriamento, e na redução do uso de água nas casas de vegetação. O controle da umidade através da ventilação pode ser usado para reduzir a umidade interna em casas de vegetação em períodos quentes, uma vez que valores de umidade acima de $90 \%$ devem ser evitados, por serem críticos no controle de doenças e pragas. Quando há uso de ventilação forçada em casas de vegetação localizadas em regiões de clima subtropical, o controle da umidade relativa do ar é mais eficiente em razão da diferença entre a umidade relativa do ar externo e o interno, sendo recomendado o uso de sistemas de resfriamento adiabático (Martinez, 1994).

O controle da umidade relativa do ar pode ser feito através dos sistemas de climatização, conforme Tawegoum et al. (2006). Os autores apresentam um sistema de controle através de um algoritmo que incorpora modelos dinâmicos que, por sua vez, representam o funcionamento de uma unidade de acondicionamento de ar. A temperatura do ar, de bulbo seco e úmido, foi medida em várias localizações da casa de vegetação. Comprovou-se a afetividade do controle da umidade relativa através da unidade de ar acondicionado, para uma ampla faixa de valores da umidade relativa, com minimização do erro do consumo de energia elétrica do sistema.

\section{Radiação solar}

O controle da radiação solar nas casas de vegetação é feito, geralmente, através de sistemas de sombreamento, com base na medição da radiação solar externa e modelos de transmissividade do material das paredes da instalação (Jewett \& Short, 1992). Malhas de controle automático de múltiplas entradas e saídas (MIMO) também têm sido usadas incorporando algoritmos e modelos lineares para o rastreamento e diagnóstico do desenvolvimento das plantas permitindo a produção em períodos que permitem maior regularização da oferta e melhor qualidade dos produtos (Sentelhas \& Santos, 1995; Sigrimis \& Rerras, 1996), porém nem todas as modificações microclimáticas são benéficas aos cultivos nas casas de vegetação, sendo um dos maiores desafios para os produtores o controle microclimático durante o verão. Durante a avaliação da produção de alface hidropônica em casas de vegetação em função da variação dos parâmetros climáticos constatou-se que, quando acionado, no sistema de resfriamento evaporativo, mesmo com altos níveis de umidade relativa do ar, o meio poroso continuava a ser umedecido sem haver necessidade, prejudicando o desenvolvimento das plantas e o aumento do consumo de energia elétrica (Costa et al., 2004).

Aplicando técnicas de simulação bi-dimensional e CFD, Montero et al. (2005), estudaram a influência da radiação solar no crescimento das plantas em casas de vegetação, fornecendo informações da temperatura do ar em diferentes horários do dia e regiões da instalação. Os autores constataram que a utilização de cortinas diminuiu significativamente a condensação de água em algumas localizações da casa, havendo potencial para o controle da temperatura interna do ar, com a instalação de forro com comportamento térmico específico para cada aplicação.

Outra opção do controle da radiação solar é o uso de coberturas de filme plástico, disponíveis no mercado em várias opções de materiais, sendo o custo de implantação relativamente baixo, e a transmissão de luz das coberturas comparável à do vidro, bloqueando e refletindo o calor radiante, favorecendo a redução do consumo de energia elétrica nas casas de vegetação, como destacado por Vischi Filho (2002).

\section{Ventilação}

O controle da vazão de ar de ventilação em casas de vegetação contribui para manter a temperatura, a umidade relativa e a concentração de $\mathrm{CO}_{2}$ dentro dos níveis recomendados, como comprovado por Khaoua et al. (2006). Referidos autores desenvolveram a simulação através de CFD, do comportamento da vazão de ar, validando os dados com medições da velocidade e da composição do ar, feitas com um anemômetro sônico tridimensional e um analisador de gases. 
Resultados de modelos de simulação em tempo real poderiam ser utilizados para se obter um sinal de atuação preciso, como mostrado por Desta et al. (2004), que desenvolveram um sistema de controle aplicando CFD e validando os resultados com dados experimentais, em que a variável de entrada era a temperatura do ar, medida em uma malha de pontos que representava fisicamente o volume total da casa de vegetação. Com a utilização desta ferramenta pode-se obter a distribuição espacial dos valores das variáveis climáticas no interior das casas de vegetação e o sistema de controle atuou mais eficientemente.

A simulação tridimensional e bi-dimensional com CFD também tem sido utilizada, através do traçando das isotermas, para modelar a influência da inclinação dos telhados e abertura das janelas, na transferência de calor; no entanto, os modelos não consideram o efeito da transpiração das plantas (Shklyar \& Arbel, 2004; Campen, 2005; Baeza et al., 2005; Brugger et al., 2005). Resultados de simulação em duas dimensões podem ser eficientes e suficientes para estudar a ventilação, segundo Molina-Aiz et al. (2005), ao comprovarem que, com o aumento das dimensões da casa de vegetação pode haver redução da eficiência da ventilação de até 88\%.

Embora a aplicação de técnicas de simulação computacional e CFD, se tenha mostrado uma ferramenta útil para o estudo, otimização e desenho de casas de vegetação, em virtude da sua complexidade, ainda não se tem aplicativos em malhas de controle de ventilação em sistemas funcionando comercialmente.

\section{Fertigação e solução nutritiva}

Estratégias imprecisas no controle da fertigação podem resultar em desequilíbrio na composição da solução aplicada às plantas, com efeito prejudicial na qualidade e rendimento das culturas, podendo trazer atrelada, também, toxicidade ou salinidade. Por outro lado, quando o controle da fertigação é realizado através da medição indireta de variáveis, como a radiação solar, e, a drenagem, surge então possibilidade de maiores erros, além de imprecisão no controle da perda de água e de sais minerais nas plantas.

Buscando minimizar os efeitos negativos do controle inadequado da fertigação, Rijck et al. (1998) propuseram um sistema que foi aplicado à cultura de tomate em casas de vegetação, o qual se baseia na determinação da condutividade térmica da lã de rocha, medindo-se o teor de umidade da mesma. Como a condutividade térmica de um substrato homogêneo aumenta com o aumento do teor de umidade, o acionamento das bombas é feito em função de um teor de sua umidade mínimo e máximo previamente definido. Os autores destacam que o sistema poderia ser utilizado para outros tipos de substratos com precisão adequada.

O controle da fertigação ainda pode ser feito pelo monitoramento da composição da água de drenagem, haja vista que os mecanismos de absorção pela planta, de cada nutriente, são diferentes e dependem do crescimento da planta e das condições climáticas; por outro lado, a variação da composição da solução nutritiva pode comprometer o reúso, uma vez que elas deverão ser reabastecidas com doses de nutrientes e água na proporção certa (Savvas, 2002).
Constata-se a proposta de sistemas de controle automático envolvendo a medição, o monitoramento e o ajuste em tempo real da composição da solução de drenagem para reúso. Uma solução adotada é a medição da condutividade elétrica da solução e ajuste automático, visando manter constante a condutividade elétrica na solução, assim como fornecer taxas de injeção padronizadas, evitando flutuações na composição. Outra solução é a injeção automática e padronizada de doses de fertilizantes na água ajustando-se, em tempo real, resultando um valor fixo da condutividade elétrica da água de irrigação (Kreij, 1995).

Uma desvantagem dessas técnicas, amplamente usadas em casas de vegetação comerciais, reside no fato de que a taxa de mistura da água de drenagem depende da condutividade elétrica e não pode ser automaticamente adaptada à proporção real de cada ciclo de irrigação; desta forma, se a proporção de drenagem for mais elevada que a taxa de mistura no processo de reúso, a descarga parcial de solução de drenagem poderá ser evitada; para superar esta limitação, o volume e a condutividade elétrica da solução de drenagem podem ser medidos após cada ciclo de irrigação, usando-os para calcular a dose necessária a se adicionar, em função da variação e da necessidade (Savvas \& Manos, 1999). No entanto, a medição em tempo real da composição de nutrientes na água de reúso em casas de vegetação, operando comercialmente, não é viável, sendo muito útil a aplicação de modelos, correlacionando a composição e a dose. Savvas (2002) propôs dois modelos alternativos para o controle, através de computador, do reabastecimento de nutrientes na solução de reúso para a fertigação, na produção de crisântemos, com base na medição da condutividade elétrica e pH da solução usada na irrigação, em função da demanda de nutrientes. A condutividade elétrica, o volume de solução drenada e o valor de $\mathrm{pH}$, são medidos durante e após o processo de mistura dos nutrientes com a água fresca. Esses dados são usados para calcular e controlar a entrada de fertilizantes mantendo-se a taxa de injeção através do controle das bombas, e dois modelos são usados para o cômputo das doses individuais de nutrientes e entrada de fertilizantes e água fresca. Os resultados mostraram que, embora ambos os modelos fossem capazes de fornecer as quantidades de nutrientes necessárias, com as devidas concentrações de $\mathrm{Ca}^{2+}, \mathrm{Mg}^{2+}$ e $\mathrm{SO}_{4}{ }^{2-}$, os problemas relacionados com a concentração excessiva de alguns elementos na água fresca podem ser controlados mas não eliminados completamente enquanto a concentração e a proporção dos elementos de potássio, cálcio e magnésio da água fresca, devem ser determinadas previamente.

O protótipo de um sistema automatizado para controle da qualidade da solução nutritiva através da medição do pH e da condutividade elétrica, durante o cultivo hidropônico de alface, foi apresentado por Machado (2000). Após a implementação e experimentação constatou-se que a medição e o controle do $\mathrm{pH}$ da solução nutritiva apresentaram menor erro quando comparados com a medição e controle da condutividade elétrica, sendo os valores do $\mathrm{pH}$ da solução nutritiva de retorno mais elevados que os valores da solução nutritiva contida no reservatório, evidenciando que a absorção de nutrientes pelas plantas altera os valores do $\mathrm{pH}$, sendo oportu- 
no, então, o controle dessas variáveis, em função das respostas da cultura. Destaca-se que, para o abastecimento de água em cultivos hidropônicos em casas de vegetação, várias linhas de irrigação são usadas, podendo haver distribuição ineficiente da vazão de água nas fileiras de plantas. Outra dificuldade é o tempo de resposta dos sensores e atuadores, notando-se atuação diferente daquela esperada no processo em função da configuração das malhas de controle, não se atingindo o equilíbrio, afetando a qualidade do processo.

Um sistema de gestão e controle como ferramenta de suporte à decisão, aplicando-se lógica fuzzy, foi apresentado por Sigrimis et al. (2000a), visando superar as deficiências dos sistemas de controle da solução nutritiva e fertigação. Propôs-se um sistema formado por um controlador lógico programável (CLP), controladores PID e blocos funcionais, em forma de livraria, programando-se várias malhas de controle. A sintonia da componente integral dos controladores PID era feita atendendo às condições iniciais do processo.

O uso de sensores mais avançados, como línguas eletrônicas, para a supervisão da composição da solução nutritiva em um sistema de fertigação, foi proposto por Gutiérrez et al. (2007). Este novo enfoque do controle da fertigação propõe a utilização de um arranjo de sensores potenciométricos, compostos por uma membrana de polímeros, acoplados a uma ferramenta de calibração multivariável, em que o processamento das respostas é realizado através de uma rede neural também multivariável. O modelo prediz a concentração de potássio, amônia e nitrato, assim como as concentrações indesejadas de sódio e cloro, na solução de drenagem e de água fresca, partindo das informações da língua eletrônica, e dos valores medidos de temperatura da solução. Os resultados mostraram a precisão das respostas, com exceção do cloro, que é bastante influenciado pela concentração de nitrato.

\section{CONCLUSÕES}

1. Constata-se avanços nas pesquisas, no uso da eletrônica, tecnologias de informação e de controle automático em casas de vegetação, visando à qualidade e eficiência da produção, no âmbito da Agricultura de Precisão.

2. Porém, percebe-se ainda distanciamento entre os resultados das pesquisas e a aplicação em sistemas comerciais em operação, relacionado com limitações operacionais das tecnologias.

3. Na medida em que as exigências do mercado por alimento seguro aumentam a aplicação de tecnologias na agricultura, e, especificamente na produção em casas de vegetação, deverá aumentar, procurando estabelecer adequada relação custo-benefício, integrando todas as etapas da cadeia produtiva, desde o plantio até o consumidor.

\section{LITERATURA CITADA}

Arvanitis, K. G.; Paraskevopoulos, P. N.; Vernardos, A. A. Multirate adaptive temperature control of greenhouses. Computers and Electronics in Agriculture, v.26, p.303-320, 2000.
Baeza, E. J.; Perez-Parra, J.; Monteiro, J. I. Effect of ventilator size in natural ventilation in parral greenhouse by means of CFD simulations. Acta Horticulturae, v.691, p.465-472, 2005.

Bartzanas, T.; Boulard, T.; Kittas, C. Effect of vent arrangement on windward ventilation of a tunnel greenhouse. Biosystems Engineering, v.88, p.479-490, 2004.

Beltrão, N. de M.; Fideles, J. F.; Figueiredo, I. Uso adequado de casa-de-vegetação e de telados na e experimentação agrícola. Revista Brasileira de Engenharia Agrícola e Ambiental, v.6, n.3, p.547-552, 2002.

Bliska, Jr, A.; Honório, S. L. Cartilha tecnológica: Plasticultura e estufas. Campinas: FEAGRI/UNICAMP, 1996. 85p.

Brugger, M.; Montero, J.; Baezz, E.; Perez-Parra, J. Computational fluid dynamic modelling to improve to design of the Spanish parral style greenhouse. Acta Horticulturae, v.691, p.425-432, 2005.

Campen, J. B. Greenhouse design applying CFD for Indonesian conditions. Acta Horticulturae, v.691, p.419-424, 2005.

Cansado, A.; Saraiva, A. M. Agrilogic: Sistema experimental para controle climático de casas de vegetação. In: Congresso da Sociedade Brasileira de Informática Aplicada à Agropecuária e à Agroindústria, 4, 2003, Porto Seguro. Sociedade Brasileira de Informática Aplicada à Agropecuária e Agroindústria, v.2. p.209-212, 2003.

Coelho, J. P.; Oliveira, P. B.; Cunha, B. J. Greenhouse air temperature predictive control using the particle swarm optimization algorithm. Computers and Electronics in Agriculture, v.49, p.330-344, 2005.

Cohen, M.; Save, R.; Biel, C.; Marfa, O. Simultaneous measurements of water stress with LVDT sensors and electrotensiometers: Application in pepper plants grown in two types of perlites. Acta Horticulturae, v.421, p.193-199, 1998.

Costa, E.; Leal, P. A. M. ; Carmo, J., Ruy R. . Modelo de simulação da temperatura e umidade relativa do ar no interior de estufa plástica. Engenharia Agricola, v.24, n.1, p.57-67, 2004.

Desta, T. Z.; Janssens, K.; Van Brecht, A.; Meyers, J.; Baelmans, M.; Berckmans, D. CFD for model-based controller development. Building and Environment, v.39, n.6, p.621-633, 2004.

El Ghoumari, M.Y.; Tantau, H. J. Serrano, J. Non-linear constrained MPC: Real-time implementation of greenhouse air temperature control. Computers and Electronics in Agriculture, v.49, p.345-356, 2005.

Gutiérrez, M.; Alegret, S.; Cáceres, R.; Casadesús, J.; Marfa, O. Valle del, M. Application of a potentiometric electronic tongue to fertigation strategy in greenhouse cultivation. Computers and Electronics in Agriculture, v.57, p.12-22, 2007.

Helmer, T.; Ehret, L.; Bittman, S. CropAssist, an automated system for direct measurement of greenhouse tomato growth and water use. Computers and Electronics in Agriculture, v.48, p.198-215, 2005.

Herrero, J. M.; Blasco, X.; Martínez, M.; Ramos, C.; Sanchis, J. Robust identification of non-linear greenhouse model using evolutionary algorithms. Control Engineering Practice, v.16, n.5, p.525-530, 2007.

Jewett, T. J.; Short, T. H. Computer control of a five-stage greenhouse shading system. St Joseph: American Society Agricultural Engineers, 1992. 256p. 
Jin, J. Y.; Hong, S. H. Online measurement and control of plant growth-development of $\mathrm{CO}_{2}$ control algorithm. St Joseph: ASAE, 1993. 25p.

Khaoua, S. A. O.; Bournet, P. E.; Migeon, C.; Boulard, T.; Chassereriaux, G. Analysis of greenhouse ventilation based on computational fluid dynamics. Biosystems Engineering, v.95, n.1, p.83-98, 2006.

Kreij, C. de. Latest insights into water and nutrient control in soilless cultivation. Acta Horticulturae, v.408, p.47-61, 1995.

Linker, R.; Gutman, P. O.; I. Seginer, I. Robust model-based failure detection and identification in greenhouses. Computers and Electronics in Agriculture, v.26, p.255-270, 2000.

Machado, P. P. Sistema computadorizado para o monitoramento de fatores ambientais e controle da qualidade da solução nutritiva no cultivo hidropônico em casas de vegetação. Rio de Janeiro: UFRRJ, 2000. 384p. Tese Doutorado

Martinez, P. F. The influence of environment conditions of mild winter climate on the physiological behavior of protected crops. Acta Horticulturae, v.357, p.29-48, 1994.

Molina-Aiz, F. D.; Valera, D. L.; Pena, A. A.; Gil, J. A. Optimization of Almeria-type greenhouse ventilation performance with computational fluid dynamics. Acta Horticulturae, v.691, p.433-440, 2005.

Montero, J. I.; Muñoz, P.; Anton, A.; Iglesias, N. Computational fluid dynamic modelling of night-time energy fluxes in unheated greenhouses. Acta Horticulturae, v.691, p.403-409. 2005.

NGMA - National Greenhouse Manufacturers Association. Environmental. http://www.ngma.com/standardpdf/Environmental.pdf. 05 Nov 2007.

Norton, T.; Sun, D. W.; Grant, J.; Fallon, R.; Dodd, V. Applications of computational fluid dynamics (CFD) in the modeling and design of ventilation systems in the agricultural industry: A review. Bioresource Technology, v.98, p.2386-2414, 2007.

Piñón, S.; Camacho, E. F.; Kuchen, B.; Peña, M. Constrained predictive control of a greenhouse. Computers and Electronics in Agriculture, v.49, p.317-329, 2005.

Reichrath, S.; Davies, T. W. Computational fluid dynamics simulations and validation of the pressure distribution on the roof of a commercial multi-span Venlo-type glasshouse. Journal of Wind Engineering and Industrial Aerodynamics, v.90, p.139-149, 2002.

Rijck, G. de; Schrevens, E.; Baerdemaker, J. de. Thermal conductivity sensing for on-line monitoring and control of the moisture content in rockwool slabs. Scientia Horticulturae, v.74, p.307-312, 1998.

Roy, J. C.; T. Boulard, T.; Kittas, C.; Wang, S. Convective and ventilation transfers in greenhouses, Part 1: The greenhouse considered as a perfectly stirred tank. Biosystems Engineering, v.83, n.1, p.1-20, 2002.
Savvas, D. Automated replenishment of recycled greenhouse effluents with individual nutrients in hydroponics by means of two alternative models. Biosystems Engineering, p.83, n.2, p.225-236, 2002.

Savvas, D.; Manos, G. Automated composition control of nutrient solution in closed soilless culture systems. Journal of Agricultural Engineering Research, v.73, p.29-33, 1999.

Sentelhas, P. C.; Santos, A. O. Cultivo protegido: Aspectos microclimáticos. Revista Brasileira de Horticultura Ornamental, v.1, n.2, p.108-115, 1995.

Setiawan, A.; Albright, L. D.; Phelan, R. M. Application of pseudo-derivative-feedback algorithm in greenhouse air temperature control. Computers and Electronics in Agriculture, v.26, p.283-302, 2000.

Shklyar, A.; Arbel, A. Numerical model of the three-dimensional isothermal flow patterns and mass fluxes in a pitched-roof greenhouse. Journal of Wind Engineering and Industrial Aerodynamics, v.92, p.1039-1059, 2004.

Sigrimis, N.; Anastasiou, A.; Rerras, N. Energy saving in greenhouses using temperature integration: A simulation survey. Computers and Electronics in Agriculture, v.26, p.321-341, 2000b.

Sigrimis, N.; Arvanitis, K. G. Pasgianos; G. D. Synergism of high and low level systems for the efficient management of greenhouses. Computers and Electronics in Agriculture, v.29, p.21-39, 2000a.

Sigrimis, N.; King, R. Advances in greenhouse environment control. Computers and Electronics in Agriculture, v.26, p.217-219, 2000.

Sigrimis, N.; Rerras, N. A linear model for greenhouse control. St Joseph: Transactions of the ASAE, 1996. 20p.

Stanford, J. V. Implementing precision agriculture in the 21st Century. Journal Agriculture. Engineering Research, v.76, p.267-275, 2000.

Straten, G.; H. Challa, H.; Buwalda, F. Towards user accepted optimal control of greenhouse climate. Computers and Electronics in Agriculture, v.26, p.221-238, 2000.

Tawegoum, R.; Teixeira, R.; Chasseriaux, G. Simulation of humidity control and greenhouse temperature tracking in a growth chamber using a passive air conditioning unit. Control Engineering Practice, v.14, p.853-861, 2006.

Trigui, M.; Gauthier, L.; Barrintong, S. Measuring and modeling greenhouse mass balance. St Joseph: American Society Agricultural Engineers, 1993. 17p.

Vischi Filho, O. J. Avaliação de casas de vegetação para fins quarentenários de flores com diferentes graus de automação. Campinas: UNICAMP, 2002. 151p. Dissertação Mestrado

Zazueta, F. S.; Bucklin, R.; Jones, P. H.; Smajstrla, A.G. Basic concepts in environmental computer control of agricultural systems. Florida: University of Florida/Cooperative Extension Services. 1991, 8p. Circular 1029. 